\title{
Daytime sleepiness and polysomnographic variables in sleep apnoea patients
}

\author{
O. Mediano*, A. Barceló\#, M. de la Peña\#, D. Gozal", A. Agustí\# and F. Barbé*
}

ABSTRACT: Excessive daytime sleepiness (EDS) is not invariably present in patients with obstructive sleep apnoea syndrome (OSAS). The aim of the present study was to investigate polysomnographic determinants of EDS in patients with OSAS.

EDS was assessed using the Epworth Sleepiness Scale (ESS) and the multiple sleep latency test (MSLT). Patients showed EDS whenever the ESS score was $>10$ and the MSLT score $<5$ min. Absence of EDS was defined as having an ESS score of $<10$ and an MSLT score of $>10 \mathrm{~min}$.

In total, 23 male patients with EDS (mean \pm SD ESS and MSLT score $17 \pm 3$ and $4 \pm 1$ min, respectively) and 17 without EDS (ESS and MSLT score $5 \pm 2$ and $16 \pm 3 \mathrm{~min}$, respectively), were studied. Both groups exhibited a similar apnoea/hypopnoea index $(62 \pm 18$ versus $60 \pm 20$ events $\left.\cdot h^{-1}\right)$. Patients with EDS exhibited shorter sleep latency (11 \pm 16 versus $18 \pm 18 \mathrm{~min})$ and greater sleep efficiency $(90 \pm 7$ versus $82 \pm 13 \%)$ than those without EDS. Patients with EDS showed lower oxygenation (lowest arterial oxygen saturation $69 \pm 12$ versus $79 \pm 8 \%$; mean arterial oxygen saturation $87 \pm 6$ versus $90 \pm 5 \%$ ). Sleep stage distribution and arousal index did not differ between the groups.

Patients with obstructive sleep apnoea syndrome and excessive daytime sleepiness are characterised by shorter sleep latency, increased sleep efficiency and worse nocturnal oxygenation than those without excessive daytime sleepiness. Nocturnal hypoxaemia can be a major determinant of excessive daytime sleepiness in patients with obstructive sleep apnoea syndrome.

KEYWORDS: Daytime sleepiness, Epworth scale, hypoxaemia, multiple sleep latency test, sleep apnoea

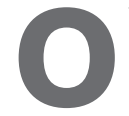
bstructive sleep apnoea syndrome (OSAS) is characterised by repeated episodes of upper airway obstruction during sleep, nocturnal hypoxaemia and sleep fragmentation [1]. Excessive daytime sleepiness (EDS) is frequently, but not universally, present in patients with OSAS. When present, EDS imposes a substantial burden on quality of life, morbidity and mortality in patients with OSAS [2] because it is a known risk factor for motor vehicle [3,4] and work-related accidents, and can result in significant psychological and cognitive deficits $[5,6]$.

The mechanisms whereby some patients with OSAS complain of EDS, whereas others do not, are unclear. Some authors have related it to the abnormal sleep structure of these patients (as assessed by the number of arousals during sleep) $[2,7,8]$, but this has not been confirmed by others [9]. Likewise, studies that investigated a potential relationship between EDS and several indices of nocturnal oxygenation also yielded controversial findings $[10,11]$.
The aim of the present study was to investigate potential mechanisms of EDS in patients with OSAS. Contrary to previous studies, however, no attempt was made to establish relationships between all of these variables in patients with varying degrees of EDS. Instead, several polysomnographic variables were compared in two groups of patients demonstrating either the absolute absence or clear presence of EDS. It was reasoned that excluding patients with intermediate levels of EDS might help to unravel the nocturnal determinants of EDS in OSAS.

\section{METHODS}

\section{Subjects}

Consecutive male patients $(n=65)$ who attended the sleep unit of the Respiratory Dept of Son Dureta University Hospital (Palma de Mallorca, Spain) and were found, by full polysomonography, to have an apnoea/hypopnoea index (AHI) of $>20$ events $\cdot h^{-1}$ were initially included in the present study. In these subjects, the presence of EDS was diagnosed by considering the results of
AFFILIATIONS

*Respiratory Dept, Arnau de Vilanova University Hospital, Biomedical Research Institute of Lleida (IRBLLEIDA), Lleida, and \#Respiratory and Biochemistry Depts, Son Dureta University Hospital, Caubet-Cimera Foundation, Palma de Mallorca, Spain. -Division of Pediatric Sleep Medicine, University of Louisville, Louisville, KY, USA.

CORRESPONDENCE

F. Barbé, Respiratory Dept, Arnau de Vilanova University Hospital, Biomedical Research Institute of Lleida (IRBLLEIDA), C/ Rovira Roure 80, 25198 Lleida, Catalunya, Spain. Fax: 34973705273

E-mail: fbarbe@arnau.scs.es

Received:

January 232006

Accepted after revision:

March 042007

SUPPORT STATEMENT

The present study was supported by grants from the Fondo de Investigaciones Sanitarias (Madrid, Spain; 02/0334, 04/1593, CM0300049 and $0300022^{\star}$ ), and by Red Respira (Madrid, Spain; RTIC 03/11), and the National Institutes of Health (Bethesda, MD, USA; HL62570), The Children's Foundation Endowment for Sleep Research and the Commonwealth of Kentucky Challenge for Excellence Trust Fund (Louisville, KY, USA) to D. Gozal.

STATEMENT OF INTEREST None declared.

European Respiratory Journal Print ISSN 0903-1936 Online ISSN 1399-3003 
both the Epworth Sleepiness Scale (ESS) and the multiple sleep latency test (MSLT; see methodological details below). EDS was considered present whenever the ESS score was $>10$ and the MSLT score $<5 \mathrm{~min}$. Conversely, absence of EDS was diagnosed in those patients with an ESS score of $<10$ and an MSLT score of $>10 \mathrm{~min}$. Of the 65 patients initially included, 25 had to be excluded due to discrepancies between ESS and MSLT results. Of the remaining 40 patients, 23 were considered to exhibit EDS and 17 not to exhibit EDS. None of the participants suffered from any chronic disease, chronic obstructive pulmonary disease, liver cirrhosis, thyroid dysfunction, rheumatoid arthritis or chronic renal failure or was taking any type of medication.

The study was approved by the Ethics and Clinical Research Committee of the Balearic Islands, and all participants signed their informed consent after being fully informed of the study goals and procedures.

\section{Polysomnographic evaluation}

The diagnosis of OSAS was established by overnight polysomnography (E-Series; Compumedics, Abbotsford, Australia). Measured variables included oronasal flow by nasal cannula, thoracoabdominal movements, ECG, submental and pretibial electromyography, electro-oculography, electroencephalography (C3/A2, C4/A1) and pulse oximetry. Apnoea was defined by the absence of airflow for $>10 \mathrm{~s}$. Hypopnoeas were defined as any airflow reduction of $>50 \%$ that lasted for $>10 \mathrm{~s}$ and resulted in either arousal or oxyhaemoglobin desaturation. An oxyhaemoglobin desaturation event was defined as a decrease in arterial oxygen saturation $\left(\mathrm{Sa}_{1} \mathrm{O}_{2}\right)$ of $>4 \%$. The $\mathrm{AHI}$ was defined as the sum of the numbers of apnoeas and hypopnoeas per hour of sleep. Sleep stages were scored using the criteria of RECHTSCHAFFEN and KALES [12]. Sleep latency was defined as the period of time between the lights being switched off and the first $30 \mathrm{~s}$ of stage 1 (sleep onset). Sleep efficiency was defined as the night sleep duration expressed as a percentage of the total sleep time in bed. Arousals were defined as recommended by the American Sleep Disorders Association Task Force criteria [13]. According to these criteria, arousals were classified as breathing-related arousals (occurring within $3 \mathrm{~s}$ following an apnoea, hypopnoea or snore) and other types of arousal (spontaneous arousal, periodic limb movementassociated arousal and technical arousal). The total number of arousals was calculated as the sum of every type of arousal.

\section{Assessment of EDS}

Subjective sleepiness was assessed using the validated Spanish version [14] of the ESS [15], a simple self-administered questionnaire, with eight-item and four-point scales that evaluate daytime somnolence among patients suffering from sleep-awake disorders. Objective sleepiness was evaluated using the MSLT according to international guidelines [16]. In brief, the MSLT consists of a series of five 20-min naps at 2-h intervals. Patients are asked to try to sleep in a dark room with a recorded montage similar to that used during polysomnography the preceding night. The mean of the individual latencies in achieving sleep during the naps is considered to represent the MSLT score. A mean sleep latency of $<5 \mathrm{~min}$ is considered to represent EDS and $>10 \mathrm{~min}$ is taken as evidence of the absence of EDS.

\section{Statistical analysis}

Results are shown as mean \pm SD. Comparisons between both groups of patients were performed using independent paired t-tests and Mann-Whitney U-tests for normally and abnormally distributed data, respectively. Correlations were performed using linear regression, followed by calculation of the Pearson correlation coefficient. Statistical significance was defined using a p-value of $<0.05$.

\section{RESULTS}

Table 1 shows the main demographic characteristics of the subjects studied. Age and body mass index (BMI) were similar in both groups. Based on the AHI, the patients suffered from severe OSAS.

Table 2 presents the main findings of the present study. Patients with EDS showed lower sleep latency and greater sleep efficiency. Furthermore, nocturnal oxygenation indices were significantly worse in the EDS group. In contrast, there were no differences in arousal indices or overall distribution of sleep stages. A significant correlation was found between MSLT score and mean nocturnal $\mathrm{Sa}_{1} \mathrm{O}_{2}(\mathrm{r}=0.36, \mathrm{p}<0.05)$, minimal nocturnal $\mathrm{Sa}_{2} \mathrm{O}_{2}(\mathrm{r}=0.47, \mathrm{p}<0.005)$ and sleep efficiency $(\mathrm{r}=-0.43, \mathrm{p}<0.005)$. Similarly the ESS score showed a significant linear correlation with the previous variables $(\mathrm{r}=-0.38, \mathrm{p}<0.05 ; \mathrm{r}=-0.5, \mathrm{p}<0.005$; and $\mathrm{r}=0.33, \mathrm{p}<0.05$, respectively).

\section{DISCUSSION}

In order to investigate potential nocturnal determinants of EDS in patients with OSAS, several polysomnographic variables were compared in two groups of patients with severe OSAS, who were carefully selected on the basis of the presence or absence of EDS (defined by the combined use of both subjective (ESS) and objective measures (MSLT)), but who were otherwise indistinguishable in terms of several potentially relevant confounding factors, such as age, sex and BMI. The main finding of the present study was that patients with EDS exhibit shorter sleep latency, greater sleep efficiency and worse nocturnal oxygenation than patients without EDS. However, there were no significant differences between the groups in AHI, arousal index and sleep macro-architecture.

The pathogenesis of EDS in patients with OSAS is unclear. In the late 1980s, GUILLEMINAULT et al. [9] did not find significant

\begin{tabular}{lccc} 
TABLE 1 & $\begin{array}{l}\text { Demographic characteristics of obstructive sleep } \\
\text { apnoea syndrome patients with and without } \\
\text { excessive daytime sleepiness (EDS) }\end{array}$ \\
& EDS & No EDS & p-value \\
\hline Subjects $\mathbf{n}$ & 23 & 17 & \\
Age yrs & $49 \pm 6$ & $50 \pm 9$ & NS \\
BMI kg $\cdot \mathbf{m}^{-2}$ & $33 \pm 5$ & $31 \pm 6$ & NS \\
Awake Sa, $\mathbf{O}_{2} \%$ & $96 \pm 1$ & $96 \pm 1$ & NS \\
ESS score & $17 \pm 3$ & $5 \pm 2$ & $<0.0001$ \\
MSLT score min & $4 \pm 1$ & $16 \pm 3$ & $<0.0001$ \\
\hline & &
\end{tabular}




\begin{tabular}{|c|c|c|c|c|}
\hline \multirow[t]{2}{*}{ TABLE 2} & \multicolumn{4}{|c|}{$\begin{array}{l}\text { Polysomnographic findings in obstructive sleep } \\
\text { apnoea syndrome patients with and without } \\
\text { excessive daytime sleepiness (EDS) }\end{array}$} \\
\hline & & EDS & No EDS & p-value \\
\hline \multicolumn{2}{|l|}{ Subjects $n$} & 23 & 17 & \\
\hline \multicolumn{2}{|l|}{ TST min } & $401 \pm 41$ & $379 \pm 63$ & NS \\
\hline \multicolumn{2}{|c|}{ Sleep latency min } & $11 \pm 16$ & $18 \pm 18$ & 0.05 \\
\hline \multicolumn{2}{|c|}{ Sleep efficiency \% } & $90 \pm 7$ & $82 \pm 13$ & 0.04 \\
\hline \multicolumn{2}{|c|}{ Awake min } & $37 \pm 29$ & $66 \pm 56$ & 0.03 \\
\hline \multicolumn{2}{|c|}{ Arousal index } & $65 \pm 20$ & $60 \pm 24$ & NS \\
\hline \multicolumn{2}{|c|}{ Respiratory arousals n } & $58 \pm 20$ & $57 \pm 22$ & NS \\
\hline \multicolumn{2}{|c|}{ AHI events $\cdot h^{-1}$} & $62 \pm 18$ & $60 \pm 20$ & NS \\
\hline \multicolumn{2}{|c|}{ Apnoea duration s } & $29 \pm 8$ & $22 \pm 7$ & 0.008 \\
\hline \multicolumn{2}{|c|}{ PLMI events $\cdot h^{-1}$} & $3 \pm 1$ & $2 \pm 1$ & NS \\
\hline \multicolumn{5}{|l|}{ Sleep \% } \\
\hline \multicolumn{2}{|l|}{ Phase 1/2 } & $81 \pm 12$ & $78 \pm 11$ & NS \\
\hline \multicolumn{2}{|l|}{ Phase 3/4 } & $6 \pm 8$ & $8 \pm 5$ & NS \\
\hline \multicolumn{2}{|l|}{ REM } & $13 \pm 6$ & $14 \pm 8$ & NS \\
\hline \multicolumn{5}{|c|}{ Nocturnal $\mathrm{Sa}, \mathrm{O}_{2} \%$} \\
\hline \multicolumn{2}{|c|}{ Minimum } & $69 \pm 12$ & $79 \pm 8$ & 0.002 \\
\hline \multicolumn{2}{|l|}{ Mean } & $87 \pm 6$ & $90 \pm 5$ & 0.01 \\
\hline
\end{tabular}

Data are presented as mean $\pm \mathrm{SD}$, unless otherwise stated. TST: total sleep time; NS: nonsignificant; AHI: apnoea-hypopnoea index; PLMI: periodic limb movement index; REM: rapid eye movement; $\mathrm{Sa}_{2} \mathrm{O}_{2}$ : arterial oxygen saturation.

associations between EDS and sleep fragmentation or nocturnal oxygenation in a large series of patients with OSAS. At about the same time, BEDARD et al. [11] suggested that EDS was related to nocturnal hypoxaemia, whereas COLT et al. [10] showed a stronger relationship between sleep fragmentation and EDS in a randomised controlled trial. Since then, several authors have indicated that EDS is primarily attributable to sleep disruption [17], whereas others have suggested that it may be related to other factors, namely alterations in oxygenation [8], or, alternatively, that indices of nocturnal hypoxaemia and sleep fragmentation contribute independently to an increased risk of hypersomnolence in patients with OSAS [18, 19]. These discrepancies between polygraphic variables and the presence of daytime somnolence prompted other investigators to develop alternative measures that may eventually improve current understanding of the mechanisms of EDS in these patients. For example, GonçALvES et al. [2] investigated the potential role of arousals during sleep and showed a significant correlation between the number of sleep-disordered-breathingrelated arousals of $>3 \mathrm{~s}$ and several subjective complaints of sleepiness (ESS) and quality of life. A recent study [8] also found that, although hypoxaemia was an important factor for the presence of daytime sleepiness, the microarousal index appeared to be the most important determining factor associated with EDS. TAUMAN et al. [20] developed a new index of sleep disruption based on the dynamic changes in spontaneous and respiratory-related arousals, which they termed sleep pressure score; a strong association was found between this score and measures of daytime neurocognitive and behavioural functioning [21]. In addition, CHERvIN et al. [22] developed a novel electroencephalographic (EEG) signal analysis method, whereby respiratory-cycle-related EEG changes were found to be useful in the prediction of EDS among patients with OSAS. Additional data on EDS and neural injury have been derived from animal models of intermittent hypoxia [23, 24], and have led to the conceptual framework proposing interactions between disease severity and duration in the context of environmentally and genetically defined susceptibility. It is not possible, of course, at this stage, to account for disease duration or the specific individual or environmental elements that may have played a role in the discrepant EDS of the present study groups; this issue must await further study.

In the present study, patients with OSAS were dichotomised to those with clear-cut EDS and those without EDS, in order to enhance potential polysomnographic differences between these two groups. Interestingly, the two groups were nonetheless similar in terms of age, BMI and disease severity, as assessed by AHI. This novel approach revealed that patients with EDS sleep more efficiently and exhibited a shorter sleep latency and a tendency to sleep longer. The present authors believe that these differences are probably a consequence (not a cause) of EDS. Furthermore, these observations suggest that patients with EDS are sleepy throughout the 24-h cycle.

EDS subjects exhibited a longer apnoea duration than those with no EDS, suggesting that there is a delay in arousal in EDS patients. The latter could either be due to intrinsic differences in arousal thresholds or may represent increased sleep pressure leading to an increased threshold for arousability. Since the severities of respiratory disturbance were similar, the AHI clearly does not provide insights into the mechanisms of EDS. Alternatively, increased hypoxaemia, leading to more severe neural damage of wake-promoting structures, may underlie the EDS [25]. It was also observed that patients with EDS showed worse nocturnal oxygenation indices than those without EDS, despite the fact that neither the AHI, arousal indices nor overall sleep architecture were significantly different between groups (table 2). These observations support, therefore, a significant role of nocturnal oxygen desaturation in the pathogenesis of EDS, as suggested previously [8, 10, 11]. Although the biological mechanisms linking nocturnal oxygen desaturation and daytime hypersomnolence in humans are unknown, the recent studies in mice of ZHAN and coworkers [26, 27] suggest that intermittent hypoxaemia during sleep triggers neural damage to brain regions that promote and control wakefulness through a convergence of oxidative and inflammatory events, which ultimately lead to neuronal cell loss and the manifestation of sleepiness [23]. The investigation of these pathways in humans and their potential use in the palliation of EDS in OSAS patients seems warranted.

In conclusion, obstructive sleep apnoea syndrome patients with excessive daytime sleepiness present with shorter sleep latency, improved sleep efficiency and worse nocturnal oxygenation than obstructive sleep apnoea syndrome patients of similar characteristics without excessive daytime sleepiness. Given that the remaining nocturnal variables investigated, including the apnoea/hypopnoea index, arousal index and sleep architecture, do not discriminate between patients with and without excessive daytime sleepiness, it was postulated that nocturnal hypoxaemia plays a major role in the pathogenesis of daytime sleepiness in patients with sleep apnoea syndrome. 


\section{REFERENCES}

1 Douglas NJ, Polo O. Pathogenesis of obstructive sleep apnoea/hypopnoea syndrome. Lancet 1994; 344: 653-655.

2 Gonçalves MA, Paiva T, Ramos E, Guilleminault C. Obstructive sleep apnea syndrome, sleepiness, and quality of life. Chest 2004; 125: 2091-2096.

3 Sassani A, Findley LJ, Kryger M, Goldlust E, George C, Davidson TM. Reducing motor-vehicle collisions, costs, and fatalities by treating obstructive sleep apnea syndrome. Sleep 2004; 27: 453-458.

4 Barbé F, Pericas J, Muñoz A, Findley L, Anto JM, Augusti AG. Automobile accidents in patients with sleep apnea syndrome. An epidemiological and mechanistic study. Am J Respir Crit Care Med 1998; 158: 18-22.

5 Munoz A, Mayoralas LR, Barbe F, Pericas J, Agusti AG. Long-term effects of CPAP on daytime functioning in patients with sleep apnoea syndrome. Eur Respir J 2000; 15: 676-681.

6 Naëgelé B, Pepin JL, Levy P, Bonnet C, Pellat J, Feuerstein C. Cognitive executive dysfunction in patients with obstructive sleep apnea syndrome (OSAS) after CPAP treatment. Sleep 1998; 21: 392-397.

7 Ciftci TU, Kokturk O, Ozkan S. Apnea-hypopnea indexes calculated using different hypopnea definitions and their relation to major symptoms. Sleep Breath 2004; 8: 141-146.

8 Miliauskas S, Sakalauskas R. Peculiarities of nocturnal oxygen saturation in obstructive sleep apnea. Medicina (Kaunas) 2005; 41: 217-220.

9 Guilleminault C, Partinen M, Quera-Salva MA, Boyd Hayes AB, Dement WC, Nino-Murcia G. Determinants of daytime sleepiness in obstructive sleep apnea. Chest 1988; 94: 32-37.

10 Colt HG, Haas H, Rich GB. Hypoxemia us sleep fragmentation as cause of excessive daytime sleepiness in obstructive sleep apnea. Chest 1991; 100: 1542-1548.

11 Bedard MA, Montplaisir J, Richer F, Malo J. Nocturnal hypoxemia as a determinant of vigilance impairment in sleep apnea syndrome. Chest 1991; 100: 367-370.

12 Rechtschaffen A, Kales A. Manual of Standardized Terminology, Techniques and Scoring System for Sleep Stages of Human Subjects. Publication No. 204. Washington, U.S. Government Printing Office, 1968.

13 EEG arousals, scoring rules and examples: a preliminary report from the Sleep Disorders Atlas Task Force of the American Sleep Disorders Association. Sleep 1992; 15: 173-184.

14 Chiner E, Arriero JM, Signes-Costa J, Marco J, Fuentes I. Validation of the Spanish version of the Epworth
Sleepiness Scale in patients with a sleep apnea syndrome. Arch Bronconeumol 1999; 35: 422-427.

15 Johns MW. A new method for measuring daytime sleepiness: the Epworth Sleepiness Scale. Sleep 1991; 14: 540-545.

16 Carskadon MA, Dement WC, Mitler MM, Roth T, Westbrook PR, Keenan S. Guidelines for the multiple sleep latency test (MSLT): a standard measure of sleepiness. Sleep 1986; 9: 519-524.

17 Gottlieb DJ, Whitney CW, Bonekat WH, et al. Relation of sleepiness to respiratory disturbance index: The Sleep Heart Health Study. Am J Respir Crit Care Med 1999; 159: 502-507.

18 Punjabi NM, O'Hearn DJ, Neubauer DN, et al. Modeling hypersomnolence in sleep-disordered breathing. A novel approach using survival analysis. Am J Respir Crit Care Med 1999; 159: 1703-1709.

19 Johns MW. Daytime sleepiness, snoring, and obstructive sleep apnea. The Epworth Sleepiness Scale. Chest 1993; 103: 30-36.

20 Tauman R, O'Brien LM, Holbrook CR, Gozal D. Sleep pressure score: a new index of sleep disruption in snoring children. Sleep 2004; 27: 274-278.

21 O'Brien LM, Tauman R, Gozal D. Sleep pressure correlates of cognitive and behavioral morbidity in snoring children. Sleep 2004; 27: 279-282.

22 Chervin RD, Burns JW, Ruzicka DL. Electroencephalographic changes during respiratory cycles predict sleepiness in sleep apnea. Am J Respir Crit Care Med 2005; 171: 652-658.

23 Gozal D, Kheirandish L. Sleepiness and neurodegeneration in sleep-disordered breathing: convergence of signaling cascades. Am J Respir Crit Care Med 2005; 171: 1325-1327.

24 Gozal D, Kheirandish L. Oxidant stress and inflammation in the snoring child: confluent pathways to upper airway pathogenesis and end-organ morbidity. Sleep Med Rev 2006; 10: 83-86.

25 Veasey SC, Davis CW, Fenik P, et al. Long-term intermittent hypoxia in mice: protracted hypersomnolence with oxidative injury to sleep-wake brain regions. Sleep 2004; 27: 194-201.

26 Zhan G, Fenik P, Pratico D, Veasey SC. Inducible nitric oxide synthase in long-term intermittent hypoxia: hypersomnolence and brain injury. Am J Respir Crit Care Med 2005; 171: 1414-1420.

27 Zhan G, Serrano F, Fenik P, et al. NADPH oxidase mediates hypersomnolence and brain oxidative injury in a murine model of sleep apnea. Am J Respir Crit Care Med 2005; 172 921-929. 